\title{
Optimization of conditions for the preparation of new adsorbent material from residues of marine algae applying a response surface methodology
}

\author{
M. Tarbaoui ${ }^{1 *}$, M. Oumam ${ }^{2}$, N. Fakhfakh ${ }^{3}$, M. Charrouf ${ }^{1}$, M. Berrada ${ }^{1}$, \\ A. Bennamara ${ }^{1}$, A. Abourriche ${ }^{1}$ \\ ${ }^{1}$ Laboratoire Biomolécules et Synthèse Organique (BioSynthO), Faculté des Sciences Ben M'sik, \\ Université Hassan II, Mohammedia-Casablanca, Maroc. \\ ${ }^{2}$ Laboratoire d'Ingénierie des Matériaux, Faculté des Sciences Ben M'sik, \\ Université Hassan II-Mohammedia-Casablanca, Maroc. \\ ${ }^{3}$ Laboratoire Eau, Energie et Environnement (ENIS), Université de Sfax, Tunisie.
}

\begin{abstract}
An experimental design has been drawn up to optimize the experimental conditions of the preparation of an adsorbent material from residues of marine algae. A series of adsorbent materials have been prepared by chemical activation with phosphoric acid. Central composite design (CCD) was applied to study the influence of activation temperature, activation time and percentage of phosphoric acid on the chemical activation process of adsorbent material. Two quadratic models were developed for yield of adsorbent material and methylene blue adsorption capacity using Design-Expert software NEMRODW. The models were used to calculate the optimum operating conditions for preparation of adsorbent material providing a compromise between yield and adsorption capacity of the process. The optimal conditions with $93.77 \%$ of methylene blue adsorption capacity and $66.14 \%$ of adsorbent material yield are obtained when using 150 min as activation time, $225^{\circ} \mathrm{C}$ as activation temperature and $70 \%$ as percentage of phosphoric acid.
\end{abstract}

Keywords: Adsorbent material, Chemical activation, Experimental design, Marine algae, Optimization.

\section{Introduction}

The valuation of algal biomass is considered among the most interesting in the use of the marine international programs. On the other hand, Morocco, with its double Atlantic and Mediterranean coast, is a country deeply influenced by the sea, which implies the existence of thousands of marine species [1]. Marine algae are potentially prolific sources of highly bioactive secondary metabolites that might represent useful leads in the development of new pharmaceutical agents [2].

However the residues of extraction obtained from these marine algae, which represent more than $80 \%$ of the weight of the raw material, are not exploited enough. This requires a valorization of theses residues by the elaboration of new adsorbent materials.

The preparation of adsorbent materials is influenced by many factors. For this reason a preliminary study on the effect of these factors on the preparation was carried out in order to determine the most important ones and their regions of interest. The most influential factors were found to be activation time $\left(\mathrm{X}_{1}\right)$, activation temperature $\left(\mathrm{X}_{2}\right)$ and the percentage of chemical activating agent $\left(\mathrm{X}_{3}\right)$. Thus, in the present work, we prepared a series of adsorbent materials from residues of marine algae with values of $\left(\mathrm{X}_{1}\right),\left(\mathrm{X}_{2}\right)$ and $\left(\mathrm{X}_{3}\right)$ included in the suitable range. Desirable preparation outputs based on mass yield $\left(\mathrm{Y}_{1}\right)$ and capacity of adsorption $\left(\mathrm{Y}_{2}\right)$ were considered as responses. Thus, an experimental design methodology is applied to relate the experimental conditions of the activated process with properties of adsorbent materials [3-4].

\section{II.1. Materials}

\section{Materials And Method}

The algae used in this study were collected from the Dar Bouaaza beach (Casablanca), Morocco in June 2011. All the reagents used were of analytical grade. Distilled water was used to prepare all solutions.

\section{II.2. Preparation of adsorbent material}

Prior to extraction, the marine algae were identified, washed with running water and with deionized water. The washed biomass was oven-dried at $60{ }^{\circ} \mathrm{C}$ for $24 \mathrm{~h}$, ground using an electric grinder to a particle size between 1 and $2 \mathrm{~mm}$, and extracted by methanol. The residues of extraction obtained from these marine algae are washed with deionized water, dried and impregnated with the same mass of phosphoric acid and mixed for half an hour in the ambient temperature. The obtained pastas are carried in the temperature of $120^{\circ} \mathrm{C}$ for 24 hours. The obtained materials underwent the reaction of activation in a leading electric thermolyne oven to self- 
regulation provided with a programmer of temperature, connected with the oven by a thermocouple and a special steel reactor. The domains of variation of activation temperature, activation time and percentage of chemical activated agent were defined on the univariate analysis. After activation, the adsorbent materials are washed with distilled water until all acid was eliminated, dried, ground and sifted to obtain a powder with a particle size capable of passing through a $100 \mu \mathrm{m}$ sieve.

\section{II.3. Univariate analysis}

Univariate analysis is the first step of analysis of process variables (temperature, time and chemical activating agent). The analysis is carried out with the description of a single variable and its attributes of the applicable unit of analysis. This step was used in the first stages of research, in analyzing the data at hand, before being supplemented by multivariate analysis using experimental design.

\section{II.3.1. Temperature of activation}

The weight loss behavior of the residues of marine sponges was measured by using a thermobalance (TGA- SETARAM). The sample was heated up to final temperature of $600^{\circ} \mathrm{C}$ at a heating of $10^{\circ} \mathrm{C} / \mathrm{min}$ under helium gas.

\section{II.3.2. Time of activation}

The analysis of activation time effect was defined using adsorption test of cationic dye, the methylene blue (MB), on the adsorbent materials elaborated at an activation temperature of $225^{\circ} \mathrm{C}$ for activation time than $15 \mathrm{~min}$, 45min, 75min, 105min, $135 \mathrm{~min}, 165 \mathrm{~min}, 195 \mathrm{~min}, 225 \mathrm{~min}, 300 \mathrm{~min}$ and 360min and using concentrated phosphoric acid. This latter is well known as precursors activating agent, allows the development of a large porosity in the activated material [5-7].

The change of the adsorption performance based on the activation time is determined at room temperature onto methylene blue solution of known initial concentration $(10 \mathrm{mg} / \mathrm{L})$. The adsorbent materials in particle size of less than $100 \mu \mathrm{m}$ are dispersed in $100 \mathrm{ml}$ of MB solution at the rate of $200 \mathrm{mg}$ of adsorbent per liter of MB solution. The suspensions were continuously agitated throughout the manipulation (overnight) to ensure a better contact between the pollutant in contact and the active sites of the product. The measurement of the concentration of methylene blue was determined using UV-visible spectrometer at a wavelength $\lambda=664 \mathrm{~nm}$. The capacity of adsorption (\%) was calculated based on Eq. (1).

Capacity of adsorption $(\%)=\frac{C_{0}-C_{e}}{C_{0}} \times 100$

where $\mathrm{C}_{0}$ and $\mathrm{C}_{\mathrm{e}}$ are the initial and final dye concentrations $(\mathrm{mg} / \mathrm{L})$ respectively.

\section{II.3.3. Percentage of chemical agent activating}

To minimize the production cost of adsorbent materials via the activation by the phosphoric acid. We studied the impact of this factor on the activation of residues of the algae by testing the methylene blue adsorption capacity of adsorbent materials prepared at $225{ }^{\circ} \mathrm{C}, 150 \mathrm{~min}$ and at different concentrations of phosphoric acid. The evolution of the adsorption capacity versus percentage of phosphoric acid was determined at room temperature on a solution of $\mathrm{MB}(10 \mathrm{mg} / \mathrm{L})$. The adsorbent materials in particle size of less than $100 \mu \mathrm{m}$ are dispersed in $100 \mathrm{ml}$ of BM solution at the rate of $100 \mathrm{mg}$ of adsorbent per liter of MB solution. The suspensions were agitated continuously throughout the manipulation (5 hours) to ensure a better contact between the pollutant in contact and the active sites of the product. The measurement of the concentration of MB was carried out using UV-visible spectrometer at a wavelength $\lambda=664 \mathrm{~nm}$.

\section{II.3.4. Adsorbent materiel yield}

The adsorbent material yield was calculated based on Eq. (2).

Yield $(\%)=\frac{W_{C}}{W_{0}} \times 100$

where $\mathrm{W}_{\mathrm{C}}$ is the dry weight $(\mathrm{g})$ of final activated material and $\mathrm{W}_{0}$ is the dry weight $(\mathrm{g})$ of precursor.

\section{II.4. Multivariate experimental design}

Response surface methodology (RSM) is a statistical method that uses quantitative data from appropriate experiments to determine regression model equations and operating conditions [8]. RSM is a collection of mathematical and statistical techniques for modeling and analysis of problems in which a response of interest is influenced by several variables [9]. A standard RSM design called central composite design (CCD) 
was applied in this work to study the variables for preparation of adsorbent material from residues of marine algae.

The central composite design was widely used for fitting a second-order model. By using this method, modeling is possible and it requires only a minimum number of experiments. It is not necessary in the modeling procedure to know the detailed reaction mechanism since the mathematical model is empirical. Generally, the CCD consists of a $2^{n}$ factorial runs with $2 n$ axial runs and $n_{c}$ center runs [10]. These designs consist of a $2^{n}$ factorial or fractional (coded to the usual \pm 1 notation) augmented by $2 \mathrm{n}$ axial points $( \pm \alpha, 0,0, \ldots, 0),(0$, $\pm \alpha, 0, \ldots, 0), \ldots,(0,0, \ldots, \pm \alpha)$, and $\mathrm{n}_{\mathrm{c}}$ center points $(0,0,0, \ldots, 0)[11-12]$. The center points were used to evaluate the experimental error and the reproducibility of the data. The axial points are chosen such that they allow rotatability [13], which ensures that the variance of the model prediction is constant at all points equidistant from the design center. Replicates of the test at the center are very important as they provide an independent estimate of the experimental error. Each variable is investigated at two levels. Meanwhile, as the number of factors, $\mathrm{n}$, increases, the number of runs for a complete replicate of the design increases rapidly. In this case, main effects and interactions may be estimated by fractional factorial designs running only a minimum number of experiments. Individual second-order effects cannot be estimated separately by $2^{\mathrm{n}}$ factorial designs. The responses and the corresponding parameters are modeled and optimized using ANOVA to estimate the statistical parameters by means of response surface methods [14].

Basically this optimization process involves three major steps, which are, performing the statistically designed experiments, estimating the coefficients in a mathematical model and predicting the response and checking the adequacy of the model.

$\mathrm{Y}=\mathrm{f}\left(\mathrm{X}_{1}, \mathrm{X}_{2}, \mathrm{X}_{3}, \mathrm{X}_{4} \ldots, \mathrm{X}_{\mathrm{n}}\right)$

where $\mathrm{Y}$ is the response of the system, and $\mathrm{X}_{\mathrm{i}}$ is the variables of action called factors. The goal is to optimize the response variable (Y). It is assumed that the independent variables are continuous and controllable by experiments with negligible errors. It is required to find a suitable approximation for the true functional relationship between independent variables and the response surface [15].

The experimental sequence was randomized in order to minimize the effects of the uncontrolled factors. The response was used to develop an empirical model that correlated the responses to the adsorption of methylene blue and production of adsorbent material process variables using a second-degree polynomial equation as given by the following equation [16]:

$Y=b_{0}+\sum_{i=1}^{n} b_{i} X_{i}+\sum_{i=1}^{n} b_{i i} X_{i}^{2}+\sum_{i=1}^{n} \sum_{j \succ 1}^{n} b_{i j} X_{i} X_{j}$

where $\mathrm{Y}$ is the predicted response, $\mathrm{b}_{0}$ the constant coefficient, $b_{i}$ the linear coefficients, $b_{i j}$ the interaction coefficients, $b_{\mathrm{ii}}$ the quadratic coefficients and $\mathrm{X}_{\mathrm{i}}, \mathrm{X}_{\mathrm{j}}$ are the coded values of the production and adsorption of methylene blue variables. The number of tests required for the CCD includes the standard $2^{n}$ factorial with its origin at the center, $2 \mathrm{n}$ points fixed axially at a distance; say $\alpha$ from the center to generate the quadratic terms, and replicate tests at the center; where $n_{c}$ is the number of variables. Hence, the total number of tests $(\mathrm{N})$ required for the three independent variables is:

$$
N=2^{n}+2 n+n_{c}=2^{3}+(2 \times 3)+2=16
$$

Once the desired ranges of values of the variables are defined, they are coded to lie at \pm 1 for the factorial points, 0 for the center points and $\pm \alpha$ for the axial points.

\section{II.5. Model fitting and statistical analysis}

The statistical software package Design-Expert, NEMRODW, was used for regression analysis of experimental data to fit the equations developed and also to plot response surface. ANOVA was used to estimate the statistical parameters.

\section{III.1. Univariate analysis}

\section{Results And Discussion}

The curve shown in Fig. 1 represents the evolution of the normalized mass of marine algae residues during pyrolysis in accordance with the temperature. The thermogram of marine algae residues shows two distinct losses:

The first took place between 25 and $225^{\circ} \mathrm{C}$, and corresponds to a slight loss, mainly due to the departure of water. The second loss from 225 to $600{ }^{\circ} \mathrm{C}$, which corresponds to a larger and complex loss. This loss begins at $225^{\circ} \mathrm{C}$ and reaches its maximum at about $375^{\circ} \mathrm{C}$ before ending at $600{ }^{\circ} \mathrm{C}$. It corresponds to a loss in weight, due to the departure of molecules derived from the decomposition of organic matter. This has allowed defining the experimental domain of activation temperature between $225^{\circ} \mathrm{C}$ and $375^{\circ} \mathrm{C}$. 


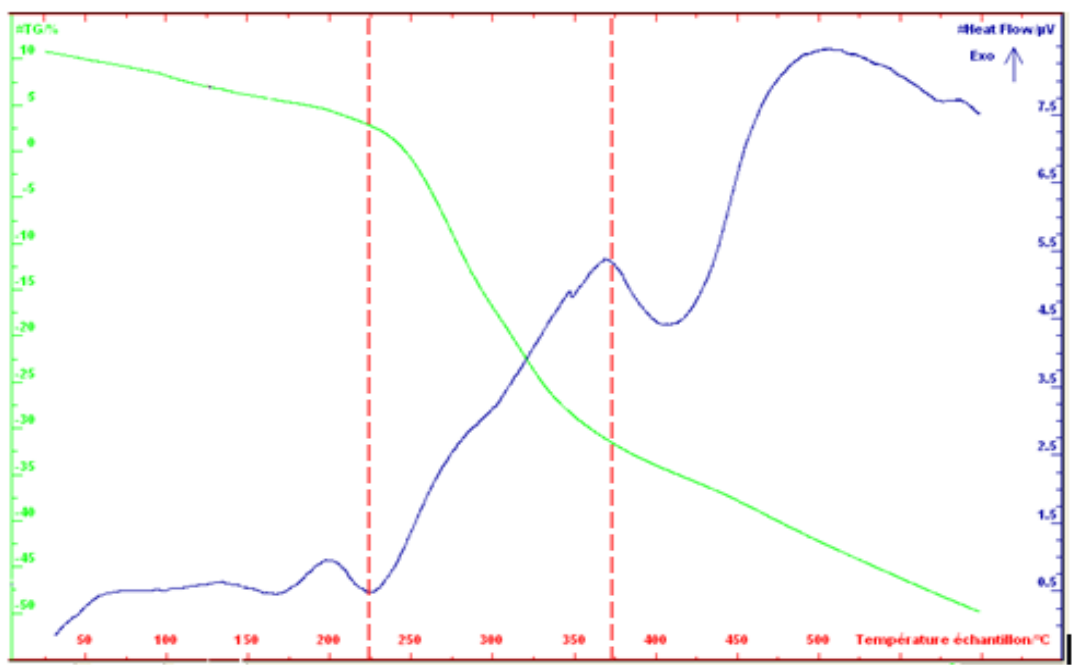

Figure 1: Thermogram of marine algae residues

\section{III.2. Time of activation}

From the Fig. 2 we find that the maximum adsorption capacity is reached from $150 \mathrm{~min}$. These results allowed us define the experimental domain of activation time which is between 15 and $150 \mathrm{~min}$.

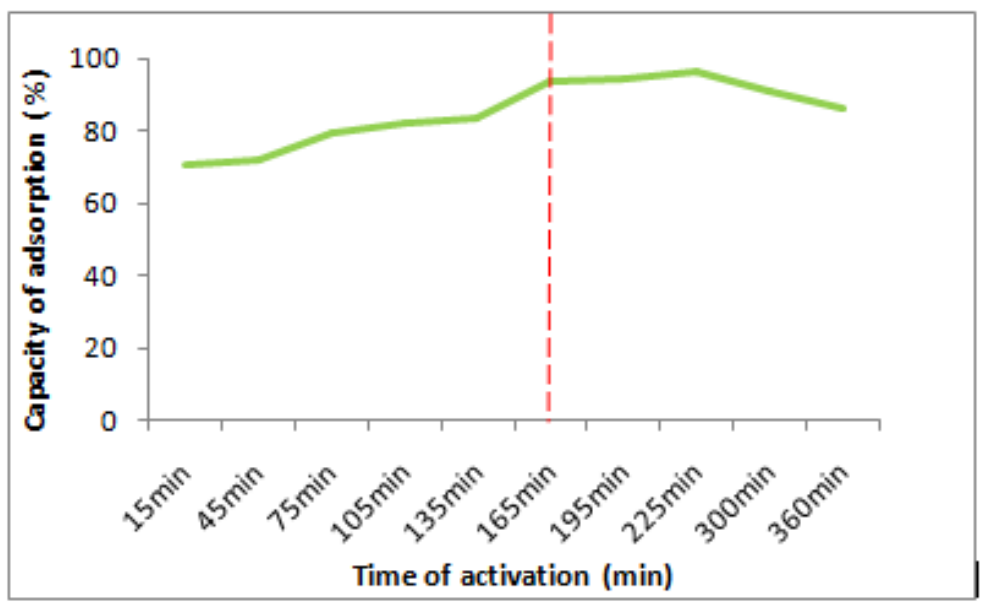

Figure 2: The effect of the activation time on the adsorption capacity of methylene blue on the elaborated materials

\section{III.3. Percentage of activating chemical agent}

From the Fig. 3 we find that the adsorbent material prepared at $225^{\circ} \mathrm{C}$ and activated with phosphoric acid at $70 \%$ is the one that gives the best adsorption capacity. These results allowed us to define the experimental field of study in the percentage of phosphoric acid which is between 10 and $70 \%$.

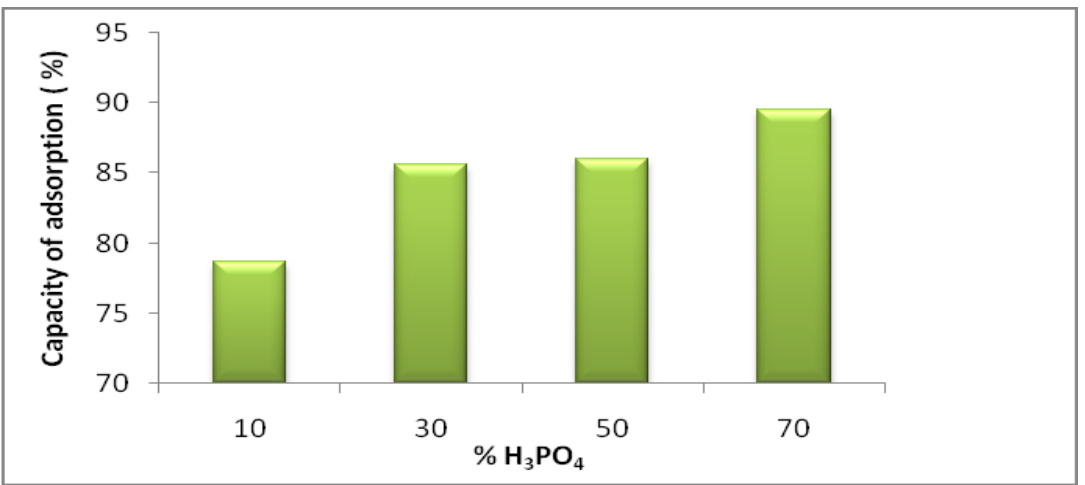

Figure 3: The effect of the percentage of $\mathrm{H}_{3} \mathrm{PO}_{4}$ on the yield and adsorption of methylene blue on the materials elaborated 
Optimization of conditions for the preparation of new adsorbent material from residues of ....

III.4. Synthesis of univariate analysis:

The univariate study allowed to determine the experimental domains for each of the three selected factors. The results are shown in Table 1.

Table 1: Experimental Domains of the factors intervener in the elaboration of adsorbent materials

\begin{tabular}{llll}
\hline Factors & Lower level $(-1)$ & central points $(0)$ & higher level $(+1)$ \\
\hline $\begin{array}{l}\text { Time of activation }\left(\mathrm{X}_{1}\right) \\
\begin{array}{l}\text { Temperature of activation } \\
\left(\mathrm{X}_{2}\right)\end{array}\end{array}$ & $15 \mathrm{~min}$ & $82,5 \mathrm{~min}$ & $150 \mathrm{~min}$ \\
$\%$ activating Agent $\left(\mathrm{X}_{3}\right)$ & $10 \%$ & $300^{\circ} \mathrm{C}$ & $375^{\circ} \mathrm{C}$ \\
\hline
\end{tabular}

\section{III.5. Development of regression model equations}

Central composite design was used to develop correlation between the adsorbent material preparation variables to the methylene blue adsorption capacity and adsorbent material yield. Runs 15 and 16 at the center point were used to determine the experimental error. The design of this experiment is given in Table 2, together with the experimental results. Regression analysis was performed to fit the response function of methylene blue adsorption capacity (\%) and adsorbent material yield (\%). The model expressed by Eq.(4), where the variables take their coded values, represents adsorbent material yield $\left(\mathrm{Y}_{1}\right)$ and methylene blue adsorption capacity $\left(\mathrm{Y}_{2}\right)$ as a function of activation times $\left(\mathrm{X}_{1}\right)$, activation temperature $\left(\mathrm{X}_{2}\right)$, and percentage of chemical activating agent $\left(\mathrm{X}_{3}\right)$. The final empirical models in terms of coded factors after excluding the insignificant terms for adsorbent material yield $\left(\mathrm{Y}_{1}\right)$ and methylene blue adsorption capacity $\left(\mathrm{Y}_{2}\right)$ are as follows: Eqs 6 and 7 .

Table 2: Experimental design matrix and results.

\begin{tabular}{|c|c|c|c|c|c|c|c|c|}
\hline \multirow[t]{2}{*}{ Run } & \multicolumn{3}{|c|}{ Coded level } & \multicolumn{3}{|c|}{ Actual level of variables } & \multirow{2}{*}{$\begin{array}{l}\text { Adsorbent } \\
\text { material yield, } \\
Y_{1}(\%)\end{array}$} & \multirow{2}{*}{$\begin{array}{l}\text { Capacity of } \\
\text { adsorption, } \\
Y_{2}(\%)\end{array}$} \\
\hline & $\mathrm{X}_{1}$ & $\mathrm{X}_{2}$ & $\mathrm{X}_{3}$ & $\mathrm{X}_{1}(\mathrm{~min})$ & $\mathrm{X}_{2}\left({ }^{\circ} \mathrm{C}\right)$ & $\mathrm{X}_{3}(\%)$ & & \\
\hline 1 & -1 & -1 & -1 & 15 & 225 & 10 & 46.40 & 90.91 \\
\hline 2 & +1 & -1 & -1 & 150 & 225 & 10 & 45.40 & 93.30 \\
\hline 3 & -1 & +1 & -1 & 15 & 375 & 10 & 48.40 & 87.44 \\
\hline 4 & +1 & +1 & -1 & 150 & 375 & 10 & 23.60 & 83.41 \\
\hline 5 & -1 & -1 & +1 & 15 & 225 & 70 & 40.00 & 94.03 \\
\hline 6 & +1 & -1 & +1 & 150 & 225 & 70 & 67.40 & 93.98 \\
\hline 7 & -1 & +1 & +1 & 15 & 375 & 70 & 66.40 & 92.22 \\
\hline 8 & +1 & +1 & +1 & 150 & 375 & 70 & 68.00 & 82.67 \\
\hline 9 & $-\alpha$ & 0 & 0 & 15 & 300 & 40 & 49.50 & 76.80 \\
\hline 10 & $+\alpha$ & 0 & 0 & 150 & 300 & 40 & 60.00 & 66.08 \\
\hline 11 & 0 & $-\alpha$ & 0 & 82.5 & 225 & 40 & 53.60 & 78.40 \\
\hline 12 & 0 & $+\alpha$ & 0 & 82.5 & 375 & 40 & 64.00 & 66.59 \\
\hline 13 & 0 & 0 & $-\alpha$ & 82.5 & 300 & 10 & 41.20 & 90.00 \\
\hline 14 & 0 & 0 & $+\alpha$ & 82.5 & 300 & 70 & 51.40 & 90.80 \\
\hline 15 & 0 & 0 & 0 & 82.5 & 300 & 40 & 57.60 & 63.96 \\
\hline 16 & 0 & 0 & 0 & 82.5 & 300 & 40 & 57.10 & 64.15 \\
\hline
\end{tabular}

$\mathrm{Y}_{1}=55.819+1.370 \mathrm{X}_{1}+1.760 \mathrm{X}_{2}+8.820 \mathrm{X}_{3}-0.303 \mathrm{X}_{1} \mathrm{X}_{1}+3.747 \mathrm{X}_{2} \mathrm{X}_{2}-8.753 \mathrm{X}_{3} \mathrm{X}_{3}$

- 6.200X $\mathrm{X}_{2}+6.850 \mathrm{X}_{1} \mathrm{X}_{3}+5.850 \mathrm{X}_{2} \mathrm{X}_{3}$

$\mathrm{Y}_{2}=68.600-2.197 \mathrm{X}_{1}-3.829 \mathrm{X}_{2}+0.864 \mathrm{X}_{3}+0.566 \mathrm{X}_{1} \mathrm{X}_{1}+1.622 \mathrm{X}_{2} \mathrm{X}_{2}+19.524 \mathrm{X}_{3} \mathrm{X}_{3}$

$-1.989 \mathrm{X}_{1} \mathrm{X}_{2}-0.994 \mathrm{X}_{1} \mathrm{X}_{3}+0.028 \mathrm{X}_{2} \mathrm{X}_{3}$

Positive sign in front of the terms indicates synergistic effect, whereas negative sign indicates antagonistic effect. The coefficient of the model for the response was estimated using multiple regression analysis technique included in the RSM. Fit quality of the models was judged from their coefficients of correlation and determination.

\subsection{Statistical analysis}

The quality of the models developed was evaluated based on the correlation coefficient value. The $\mathrm{R}^{2}$ values for Eqs.(6) and (7) were 0.948 and 0.942, respectively. Both the $\mathrm{R}^{2}$ values obtained were relatively high (close to unity), indicating that there was a good agreement between the experimental and the calculated values of the determination coefficients of the multilinear regression from the models.

The adequacy of the models was further justified through analysis of variance (ANOVA).

The ANOVA for the quadratic model for adsorbent material yield is listed in Table 3.

Reading the data in Table 3 reveals the validity of the model since the value of $F_{\text {exp }}$ (177.30), which is the ratio between the Lack of fit and the pure error, is much lower than the critical value of Fisher $\left(\mathrm{F}_{0.001(5.1)}=\right.$ 5764 ) at a $99.9 \%$ level of confidence with 5 and 1 degrees of freedom. 
The results of the ANOVA (Table 3) also shows that the experimental value of Scnedecor $(F \exp =12,11)$, which is the ratio between the square of the model and the average mean square of the residue, is higher than the critical value of Fisher $\left(\mathrm{F}_{0.01}(9.6)=7,98\right)$ at a $99 \%$ level of confidence with 9 and 6 degrees of freedom. Consequently, the regression is thus very significant and the model is considered corresponding.

The ANOVA for the quadratic model for methylene blue adsorption capacity is listed in Table 4. Reading the data in Table 4 reveals the validity of the model since the value of $F_{\exp } \quad(1272.44)$ is lower than the critical value of Fisher $\left(\mathrm{F}_{0.001(5.1)}=5764\right)$ at a $99.9 \%$ level of confidence with 5 and 1 degrees of freedom.

The results of the ANOVA (Table 4) also shows that the experimental value of Scnedecor $(F e x p=11537.99)$, is largely higher than the critical value of Fisher $\left(F_{0.001}(9.6)=18,69\right)$ at a $99.9 \%$ level of confidence with 9 and 6 degrees of freedom. Consequently, the regression is thus very significant and the model is considered corresponding.

The residue analysis of responses $\mathrm{Y}_{1}$ and $\mathrm{Y}_{2}$ are shown in Fig.4 and 5. The representations as cloud point depending on the value of the calculated response (Fig.4a and 5a) allow to verify that the experimental variance remains constant regardless of the response values. Indeed, the residues are randomly distributed around zero. The Henry's straight line (Fig.4b and 5b) allow to deduce that the residues follow a normal distribution; in fact, the points are almost aligned in a graph.

The overall quality of regressions is considered good with respect to the values of correlation factors $\mathrm{R}^{2}$ multilinear and to the random dispersion of residues; this latter result is illustrated by the alignment of the points representing the values of the probability as a function of residues.

Table 3: Analysis of variance (ANOVA) for response surface quadratic model for adsorbent material yield ( $\left.\mathrm{Y}_{1}\right)$.

\begin{tabular}{|c|c|c|c|c|c|}
\hline Source of variation & Sum of squares & $\begin{array}{l}\text { Degree of } \\
\text { freedom }\end{array}$ & Mean square & $\mathrm{F}_{\exp }$ & Signif \\
\hline Regression & $2.01520 \mathrm{E}+0003$ & 9 & $2.23911 \mathrm{E}+0002$ & 12.1101 & $0.420 * *$ \\
\hline Residual & $1.10938 \mathrm{E}+0002$ & 6 & $1.84897 \mathrm{E}+0001$ & & \\
\hline Lack of fit & $1.10813 \mathrm{E}+0002$ & 5 & $2.21626 \mathrm{E}+0001$ & 177.3012 & 5.9 \\
\hline Pure error & $1.25000 \mathrm{E}-0001$ & 1 & $1.25000 \mathrm{E}-0001$ & & \\
\hline Correlation total & $2.12614 \mathrm{E}+0003$ & 15 & & & \\
\hline
\end{tabular}

** Significant at a $99 \%$ level of confidence

Table 4: Analysis of variance (ANOVA) for response surface quadratic model for methylene blue adsorption

\begin{tabular}{llllr}
\hline \multicolumn{5}{c}{ capacity $\left(\mathrm{Y}_{2}\right)}$. \\
\hline Source of variation & Sum of squares & Degree of freedom & Mean square & $\mathrm{F}_{\exp }$ \\
\hline Regression & $1.83510 \mathrm{E}+0003$ & 9 & $2.03900 \mathrm{E}+0002$ & 11537.9991 \\
Residual & $1.12450 \mathrm{E}+0002$ & 6 & $1.87418 \mathrm{E}+0001$ & $1.09 *$ \\
Lack of fit & $1.12433 \mathrm{E}+0002$ & 5 & $2.24866 \mathrm{E}+0001$ & 1272.4441 \\
$\quad$ Pure error & $1.76720 \mathrm{E}-0002$ & 1 & $1.76720 \mathrm{E}-0002$ & $2.30 *$ \\
Correlation total & $1.94755 \mathrm{E}+0003$ & 15 & & \\
\hline
\end{tabular}

* Significant at a $95 \%$ level of confidence

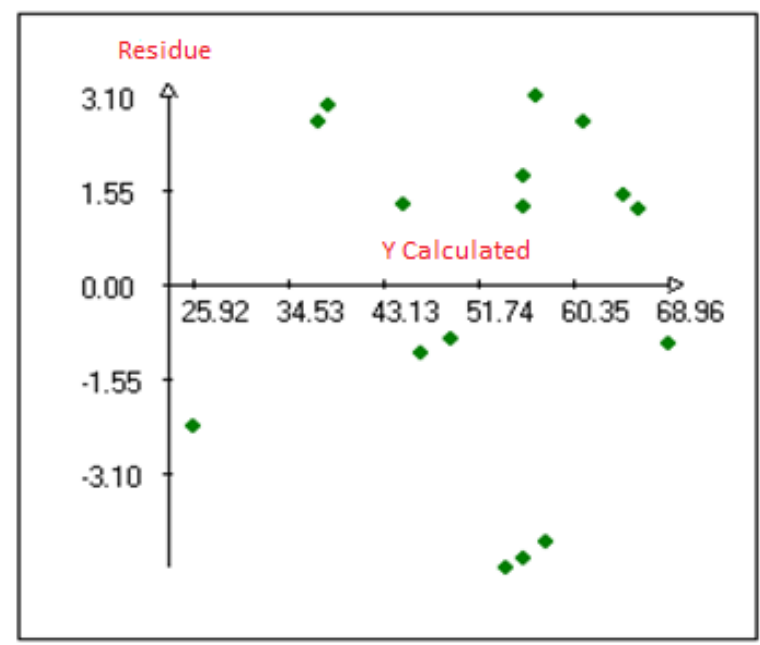

a- Graphical study of residues

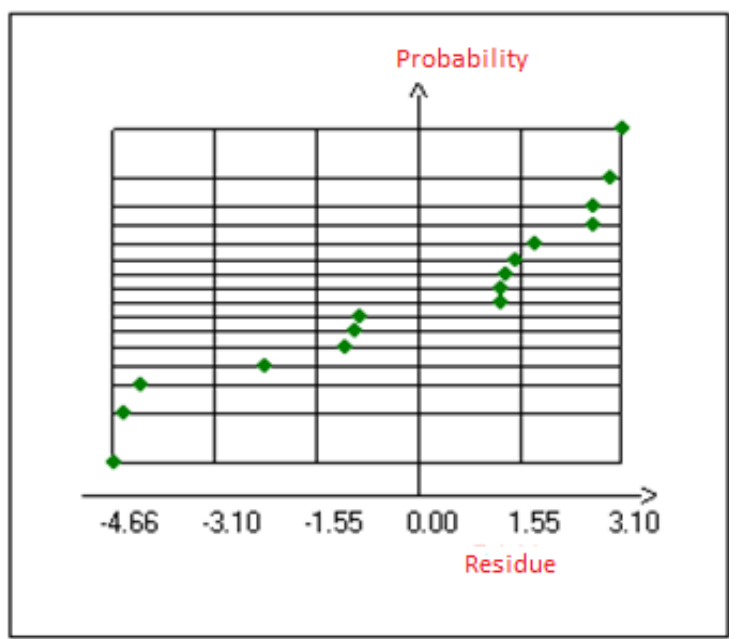

b- Henry's straight line

Figure 4: Study of residues of response $\mathrm{Y}_{1}$ (adsorbent material yield) 


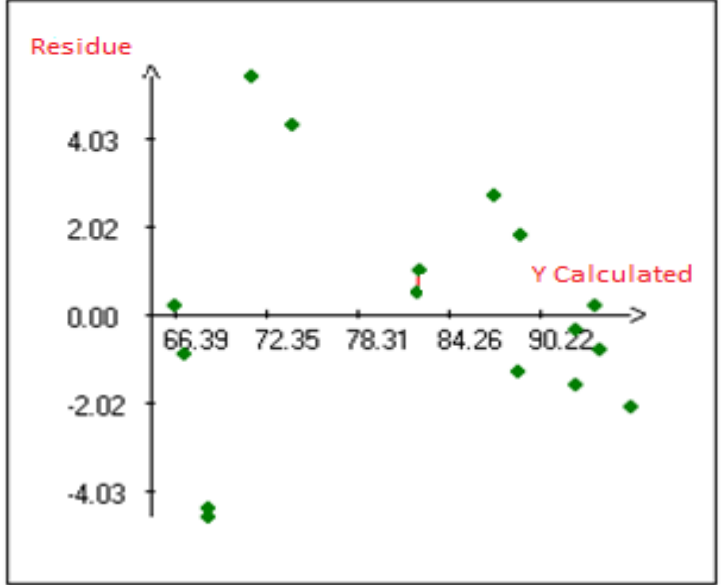

a- Graphical study of residues

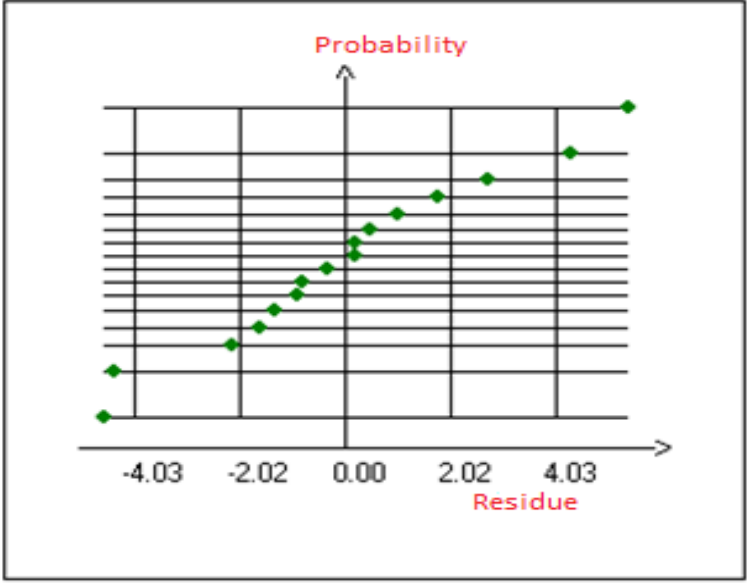

b- Henry's straight line

Figure 5: Study of residues of response $Y_{2}$ (methylene blue adsorption capacity)

\section{III.7. Adsorbent material yield}

To investigate the effects of the three factors on the Adsorbent material yield, the response surface methodology was used, and two and three-dimensional plots were drawn. The Adsorbent material yield percent response surface graphs were shown in Figs.6, 7 and 8.

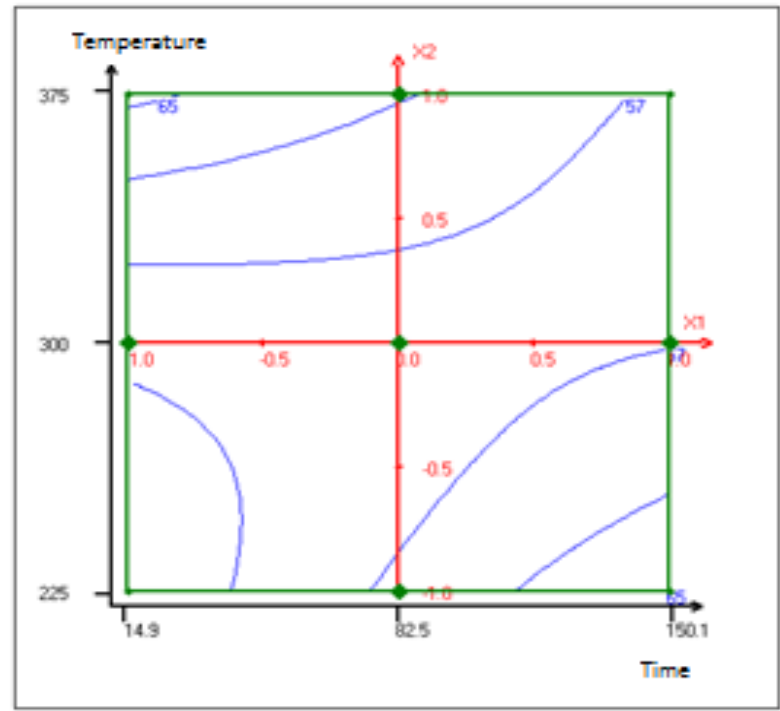

a- Graphical study at 2D

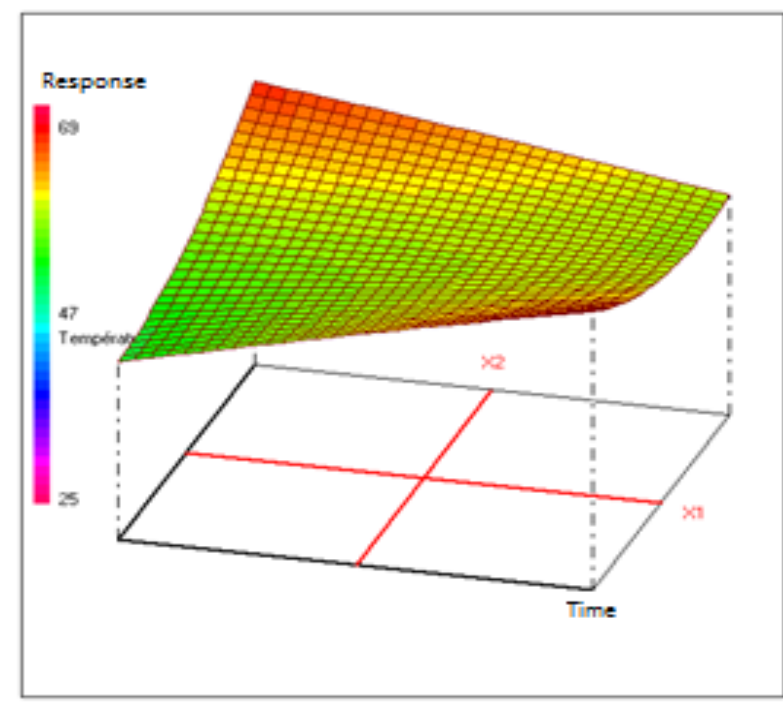

Graphical study at 3D

Figure 6: Isoresponse curves (a) and response surface (b) of adsorbent material yield: (The combined effect of activation temperature and time at constant percentage of chemical agent $(40 \%)$ 


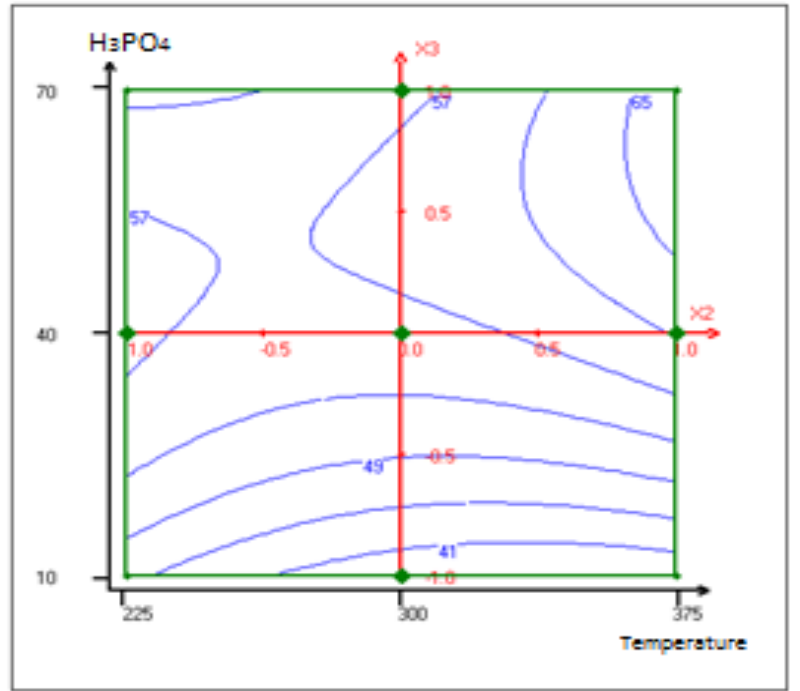

a-Graphical study at 2D

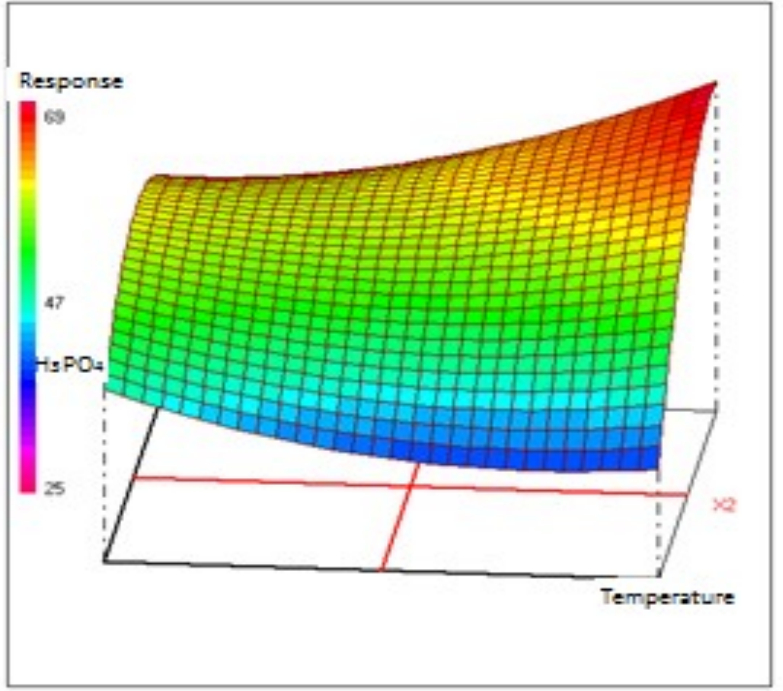

Graphical study at 3D

Figure 7: Isoresponse curves (a) and response surface (b) of adsorbent material yield: (the combined effect of activation temperature and percentage of chemical agent at constant activation time $(82,5 \mathrm{~min})$

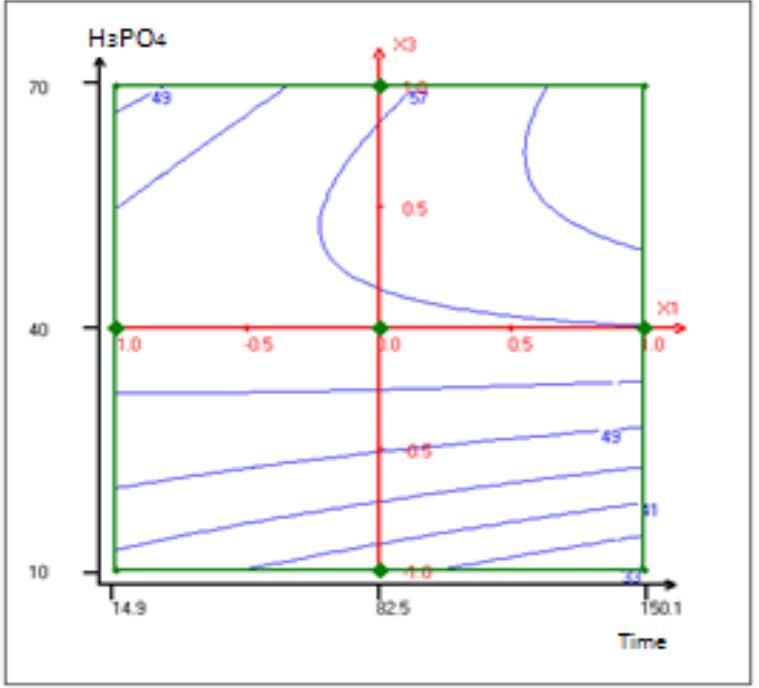

a-Graphical study at 2D

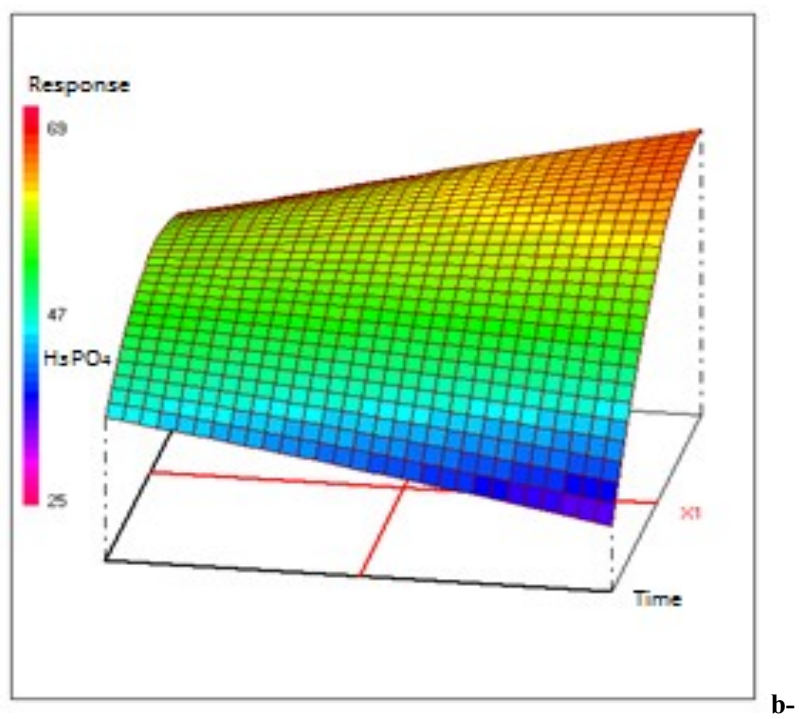

Graphical study at 3D

Figure 8 Isoresponse curves (a) and response surface (b) of adsorbent material yield: (the combined effect of activation time and percentage of chemical agent at constant activation temperature $\left(300^{\circ} \mathrm{C}\right)$

The influence of time $\left(b_{1}=1.37\right)$ on the mass yield of the adsorbent material is almost the same order as that of the temperature $\left(b_{2}=1.76\right)$. The isoresponse curves and the response surface (Fig.6) show that the maximum yield $(69 \%)$ is achieved with an activation time $=150 \mathrm{~min}$ and an activation temperature $=225^{\circ} \mathrm{C}$ or with an activation time $=15 \mathrm{~min}$ and an activation temperature $=375^{\circ} \mathrm{C}$.

The influence of percentage of chemical activating agent on adsorbent material yield $\left(\mathrm{b}_{3}=8.82\right)$ is higher than that of the temperature $\left(b_{2}=1.76\right)$. The positive sign of $b_{2}$ and $b_{3}$ proves that an increase in percentage of chemical activating agent and of the temperature results an improvement of mass yield (Fig.7). The isoresponse curves and the response surface (Fig.7) show that the highest yield (69\%) is achieved with a percentage of chemical activating agent $=70 \%$ and an activation temperature $=375^{\circ} \mathrm{C}$.

The influence of percentage of chemical activating agent on adsorbent material yield $\left(b_{3}=8.82\right)$ is higher than that of the time $\left(b_{1}=1.37\right)$. The positive sign of $b_{1}$ and $b_{3}$ proves that an increase in percentage of chemical activating agent and of the time results an improvement of mass yield (Fig.8). The isoresponse curves and the response surface (Fig.8) show that the highest yield (69\%) is achieved with a percentage of chemical activating agent $=70 \%$ and an activation time $=150 \mathrm{~min}$. 
III.8. Adsorption capacity on methylene blue

The effects of the three factors on the methylene blue adsorption were shown in Figs.9, 10 and 11.

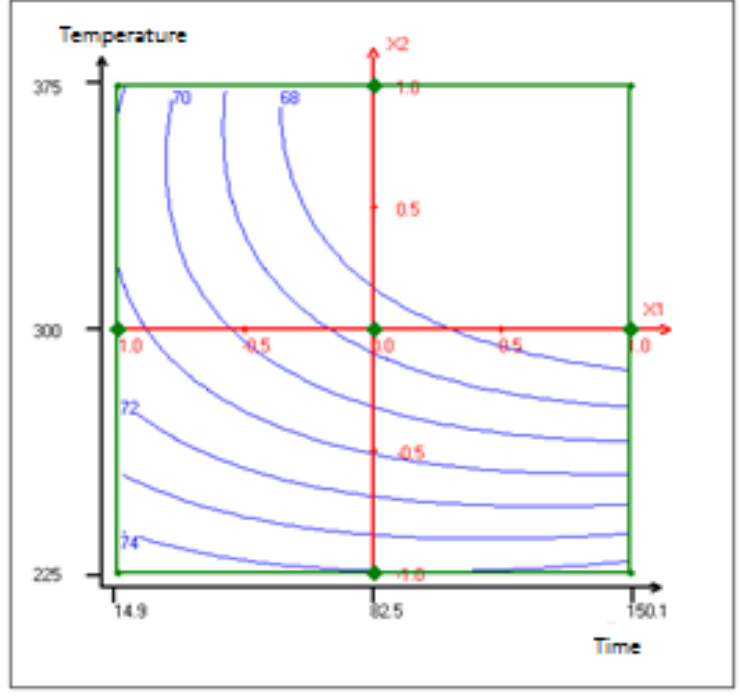

a- Graphical study at 2D

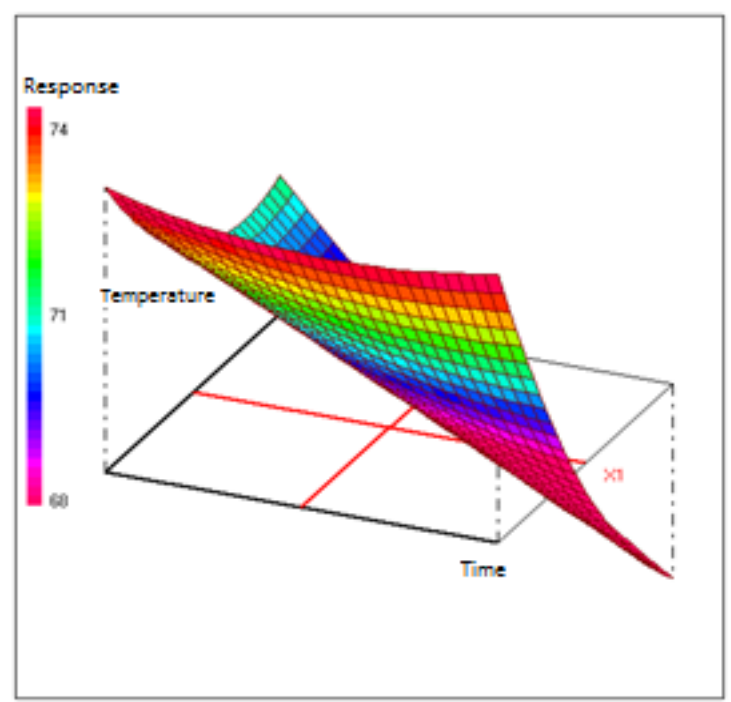

b- Graphical study at 2D

Figure 9: Isoresponse curves (a) and response surface (b) of methylene blue adsorption capacity: (The combined effect of activation temperature and time at constant percentage of chemical agent (40\%)

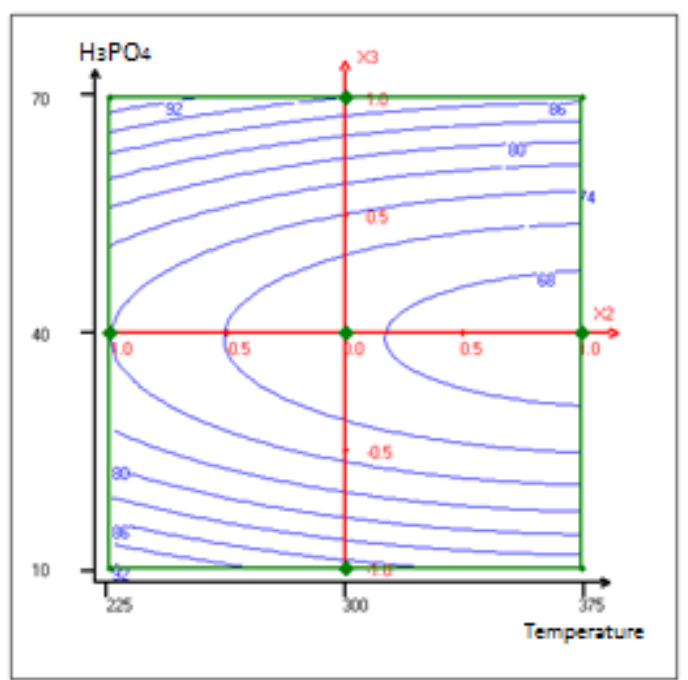

a- Graphical study at 2D

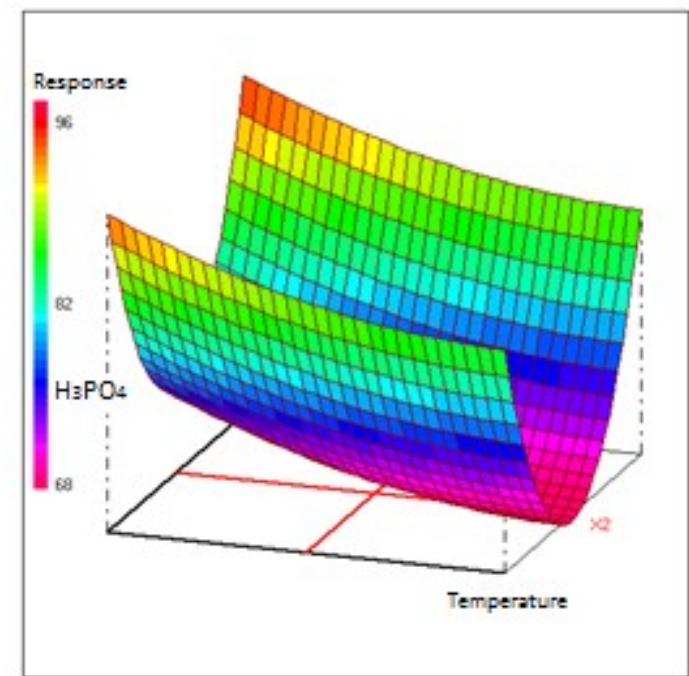

b- Graphical study at 2D

Figure 10: Isoresponse curves (a) and response surface (b) of methylene blue adsorption capacity (the combined effect of activation temperature and percentage of chemical agent at constant activation time $(82,5 \mathrm{~min})$ 


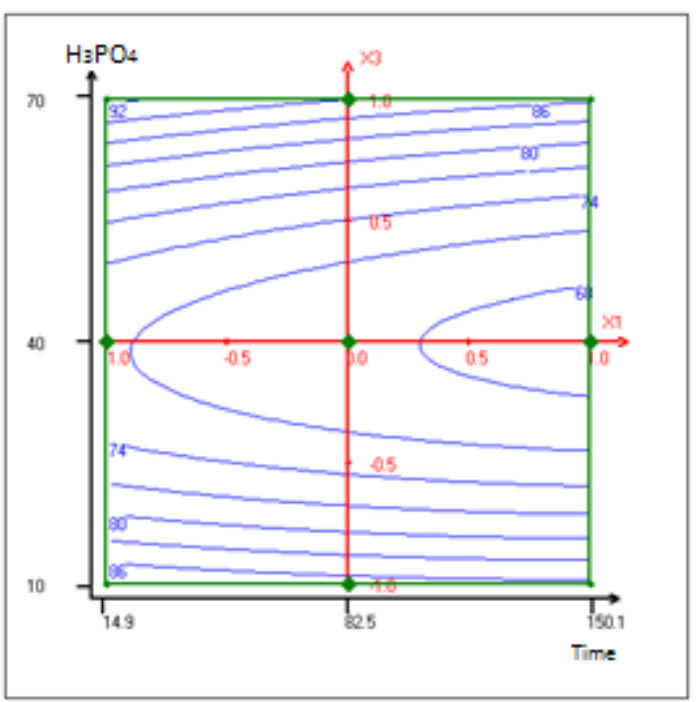

a-Graphical study at 2D

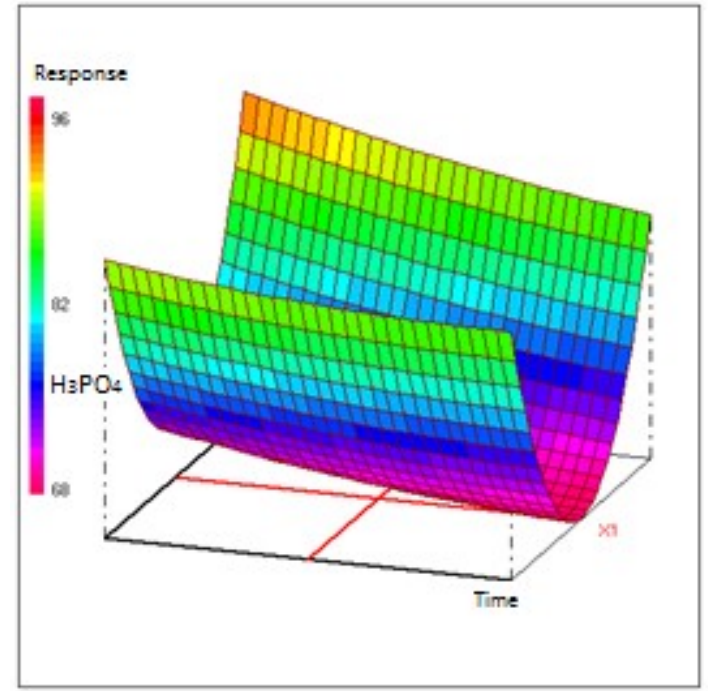

b- Graphical study at 3D

Figure 11: Isoresponse curves (a) and response surface (b) of methylene blue adsorption capacity (the combined effect of activation time and percentage of chemical agent at constant activation temperature $\left(300^{\circ} \mathrm{C}\right)$

$b_{1}$ coefficient $(-2.197)$ of the time $\left(X_{1}\right)$ in the model is more important than the temperature $\left(X_{2}\right)\left(b_{2}=-\right.$ $3.829)$, it is therefore the most influential factor on the adsorption capacity. The isoresponse curves and the response surface (Fig.9) show that the maximum adsorption capacity (74\%) is attained with an activation time between 15 and $150 \mathrm{~min}$ and an activation temperature $=225^{\circ} \mathrm{C}$.

The influence of percentage of chemical activating agent $\left(b_{3}=0.864\right)$ on the adsorption capacity is very important compared to that of the temperature $\left(b_{2}=-3.829\right)$. The negative sign of $b_{2}$ shows that a decrease in temperature results an improvement of the adsorption capacity (Fig.10). The isoresponse curves and the response surface (Fig.10) show clearly that the maximum adsorption capacity $(96 \%)$ is achieved with a percentage of chemical activating agent $=10 \%$ or $70 \%$ and an activation temperature $=225{ }^{\circ} \mathrm{C}$.

The influence of percentage of chemical activating agent $\left(b_{3}=0.864\right)$ on the adsorption capacity is very important compared to that of the time $\left(b_{1}=-2.197\right)$. The negative sign of $b_{1}$ shows that a decrease in time results an improvement of the adsorption capacity (Fig.11). The isoresponse curves and the response surface (Fig.11) show clearly that the maximum adsorption capacity (96\%) is achieved with a percentage of chemical activating agent $=70 \%$ and an activation time $=15 \mathrm{~min}$.

\section{III.9. Process optimization}

In the production of adsorbent material, one of the main aims of this study was to find the optimum process parameters relatively high product yields are expected for economical feasibility and the most important property of adsorbent material is its adsorption capacity. Therefore, the adsorbent material produced should have a high mass yield and also a high adsorption capacity for economical viability. Based on the review of all the results we find that the experience 6 is the compromise to maximize the two responses, the optimal operating conditions are presented in Table 5.

The optimal adsorbent material was obtained using preparation condition as: 150 min activation time, $225^{\circ} \mathrm{C}$ activation temperature, and $70 \%$ of $\mathrm{H}_{3} \mathrm{PO}_{4}$ : chemical activating agent, which resulted in $66.14 \%$ of mass yield and $93.77 \%$ of methylene blue adsorption capacity.

Table 5: Model validation

\begin{tabular}{lllll}
\hline $\begin{array}{l}\text { Activation time, } \\
\mathbf{X}_{\mathbf{1}}(\mathbf{m i n})\end{array}$ & $\begin{array}{l}\text { Activation } \\
\text { temperature, } \\
\mathbf{X}_{\mathbf{2}}\left({ }^{\circ} \mathbf{C}\right)\end{array}$ & $\begin{array}{l}\text { Percentage of } \\
\text { activating agent }\end{array}$ & $\begin{array}{l}\text { Adsorbent material yield, } \\
\mathbf{Y}_{\mathbf{1}}(\%)\end{array}$ & $\begin{array}{l}\text { Adsorption capacity, } \\
\mathbf{Y}_{\mathbf{2}}(\mathbf{\%})\end{array}$ \\
\hline 150 & 225 & 70 & 66.14 & 93.77 \\
\hline
\end{tabular}

\section{Conclusion}

The residues of marine algae are a good precursor for the optimization of adsorbent materials with interesting characteristics (great adsorption capacity and high mass yield).

The response surface methodology is an appropriate tool to study optimization of the activation process to prepare adsorbents materials to be used in a given technological process. 
In the present paper, this optimization was carried out to obtain adsorbent materials from marine algae residues with suitable characteristics for use in water treatments.

The optimal adsorbent material was obtained using $225^{\circ} \mathrm{C}$ activation temperature, 150 min activation time and $70 \%$ of chemical activating agent, resulting in $66.14 \%$ of mass yield and $93.77 \%$ of methylene blue adsorption capacity.

\section{References}

[1] T. Ainane, A. Abourriche, M. Kabbaj, M. Elkouali, A. Bennamara, M. Charrouf, M. Talbi, and M. Lemrani, Journal of Chemical and Pharmaceutical Research, 6(4), 2014, 607-611.

[2] A.A. El Gamal, Biological importance of marine algae, Saudi Pharmaceutical Journal, 18(1), 2010, 1-25.

[3] S. Behij, K. Djebali, H. Hammi, A.H. Hamzaoui, and A. M'nif, Optimisation of epsomite transformation into periclase using experimental design methodology, Journal of Chemometrics, 25(2), 2011, 59-66.

[4] R. Fezei, H. Hammi, and A. M'nif, Optimization of sylvite transformation into arcanite using experimental design methodology, Journal of Chemometrics, 22(2), 2008, 122-129.

[5] A. Ahmadpour, and D.D. Do, The preparation of activated carbon from Macadamia nutshell by chemical activation, Carbon, 35 (12), 1997, 1723-1732

[6] C.J. Blanco, P.R. Bonelli, E.G. Cerrella, and A.L. Cukierman, Phosphoric acid activation of agricultural residues and baggasse from sugar cane: influence of the experimental conditions on adsorption characteristics of activated carbons, Industrial \& Engineering Chemistry Research, 39 (11), 2000, 4166-4172.

[7] A.M. Puziy, O.I. Poddubnaya, A. Martinez-Alenso, F. Suarez-Garcia, and J.M.D. Tascon, Synthetic Carbons Actived with Phosphoric Acid; Surface Chemistry and Ion Binding Properties, Carbon, 40(9), 2002, 1493 - 1505.

[8] M.Z. Alam, S.A. Muyibi, and J. Toramae, Statistical optimization of adsorption processes for removal of 2,4-dichlorophenol by activated carbon derived from oil palm empty fruit bunches. Journal of Environmental Sciences, 19(6), 2007, 674-677.

[9] N. Chaudhary, and C. Balomajumder, Optimization study of adsorption parameters for removal of phenol on aluminum impregnated fly ash using response surface methodology, Journal of the Taiwan Institute of Chemical Engineers, 45(3), 2014, $852-859$.

[10] J.M. Salman, Optimization of preparation conditions for activated carbon from palm oil fronds using response surface methodology on removal of pesticides from aqueous solution, Arabian Journal of Chemistry, 7(1), 2014, 101-108.

[11] R.H. Myers, Surface Methodology (Allyn and Bacon, New York, 1971).

[12] T.J. Napier-Munn, The Central Composite Rotatable Design JKMRC, The University of Queensland, Brisbane, Australia, 2000, pg. $1-9$.

[13] G.E.P. Box, and J.S. Hunter, Multifactor experimental designs for exploring response surface, Annals Mathematical Statistics , 28(1), 1957, 195-241.

[14] O.P. Junior, A.L. Cazetta, R.C. Gomesa, É.O. Barizão, I. P.A.F. Souza, A.C. Martinsa, T. Asefa, and V.C. Almeida, Optimization using RSM and Blue Methylene Adsorption, Journal of Analytical and Applied Pyrolysis, 105, 2014, $166-176$.

[15] V. Gunaraj, and N. Murugan, Application of response surface methodology for predicting weld bead quality in submerged arc welding of pipesJournal of Materials Processing Technology, 88(1), 1999, 266-275.

[16] J. Jentzer, M. Alignan, C. Vaca-Garcia, L. Rigal, and G. Vilarem, Response surface methodology to optimise Accelerated Solvent Extraction of steviol glycosides from $<\mathrm{i}>$ Stevia rebaudiana $</ \mathrm{i}>$ Bertoni leavesFood Chemistry, 166, 2015, 561-567. 